Egyptian

Orthodontic Journal

\title{
EVALUATION OF THREE METHODS OF SURFACE TREATMENT FOR BONDING ORTHODONTIC METAL BRACKETS TO RESIN COMPOSITE IN VITRO
}

\author{
Sherif H. Darwish ${ }^{1}$, Hassan I. Abdallah², Mohamed I. Mowafy ${ }^{3}$
}

\section{ABSTRACT:}

Objective: The aim of this study is to compare shear 6ond strength of orthodontic stainless steel brackets to resin composite surfaces In vitro using three different methods of surface treatment. Materials and methods: 60 composite resin discs, $6 \mathrm{~mm}$ in diameter and $2 \mathrm{~mm}$ in height, were prepared and aged by immersion in water for 4 weeks. After ageing, the specimens were randomly assigned to one of the following groups: (1) Control with no surface treatment, (2) 99 per cent Acetone, (3) methyl methacrylate monomer, (4) Diamond bur. The metal brackets were bonded to composite surfaces by means of an orthodontic adhesive. All specimens were stored in water for 1 week. Shear Bond Strength values were tested using a universal testing machine at a crosshead speed of $1 \mathrm{~mm} /$ minute, the brackets bases and corresponding RC discs were examined under stereomicroscope at 10X magnification for adhesive remnant evaluation. Shear bond strength values were analyzed using 1-way analysis of variance (ANOVA)), Adhesive remnant index scores were analysed using Chi-square test using Monte Carlo correction. Significance of the obtained results was judged at the 5\% level ( $P \leq 0.05)$. Results: The results of 1-way ANOVA for SBS means showed no statistical significant difference among the 4 groups $(P=0.564)$. The result of Chi-square test for ARI scores between the four

1- Post-Graduate student, Orthodontic Department, Alexandria University.

2- Professor, Orthodontic Department, Alexandria University.

3- Assistant Professor, Orthodontic Department, Alexandria University. 
Egyptian

Orthodontic Journal

groups showed a statistically significant difference (MCp $=0.039$ ). Chi square comparisons of ARI scores showed statistically significant difference only between group 1 (control) with groups 3 $(\mathfrak{P} 1=0.003)$ and group $4(\mathcal{P} 1=0.046)$

\section{INTRODUCTION}

In 1965 Newman $^{1}$ introduced bonding between an orthodontic bracket and the enamel surface. Enamel is a reliable bonding substrate owing to its high inorganic content which forms a retentive pattern by acid etching. ${ }^{2}$ Current orthodontic practice requires the orthodontist to be able to bond not only enamel, but to a variety of restorative materials as The number of adults seeking adjunctive and comprehensive orthodontic treatment increased, most having a heavily restored dentition ${ }^{3,4}$. In addition, there are certain instances in which patients with anterior restorations are more likely to present at orthodontic offices. These patients would include those with severely-protruding maxillary incisors that are at considerable risk of fracture and thus requiring restoration. ${ }^{5}$ It is not uncommon, therefore, to find situations in which orthodontic brackets must be bonded to RC surfaces.

Composite restorations have posed a new challenge to both the operative dentist and orthodontist. Many studies have shown that once the $\mathrm{RC}$ has been contaminated, polished or aged, the bond strength of RC to that surface is significantly reduced. ${ }^{6 \sim 10}$ by 20 to $60 \% .^{11}$ It must be considered, however, that bonding orthodontic brackets is done for a long term temporary procedure rather than a permanent bond as required in operative dentistry. ${ }^{12}$ It has been suggested that bond strengths of 6-10 megapascals (MPa) are sufficient for orthodontic bonding. ${ }^{13,14}$

IN general, the approaches suggested to improve bond strength to such surfaces can be grouped into three broad categories: 1) mechanical by either sandblasting ${ }^{15}$ or using a coarse diamond stone. ${ }^{15}, 2$ ) chemical by etching the surface or changing the surface affinity to the adhesive materials by using a coupling agent, such as silane ${ }^{16,17}$, or 3) combination. ${ }^{18}$ Recently, Application of physical methods as gasiform ozone ${ }^{19}$ and laser ${ }^{20}$ have been also tested to boost the bond strength of brackets to resin composite surfaces. 
previous studies support the use of mechanical methods as airborne aluminum trioxide particle abrasion and roughening the composite surface with a diamond bur as it achieves a greater bond strength to composite surfaces than some chemical surface treatment as applying phosphoric acid etching and hydro-fluoric acid etching as surface treatments. However, at the expense of the surface integrity of the restoration. $^{14,15,21}$ Surface treatment with Acetone can achieve an acceptable bond strength in composite repair ${ }^{22}$ and wetting resinous surfaces with MMA monomer can achieve an acceptable SBS for adding RC to PMMA resin, ${ }^{23 ~ 25}$ both materials can be tested as surface treatments for bonding orthodontic brackets to RC surfaces.

The rationale of this study is to test other chemical methods of surface treatment and compare them to mechanical methods. In an attempt to achieve a durable bond strength during orthodontic treatment without jeopardizing the surface integrity of composite restoration.

\section{MATERIALS AND METHODS}

Sample size calculation was done using a computer program (PS: Power and Sample Size program)

\section{Composite discs Fabrication}

60 Restorative composite resin discs, $6 \mathrm{~mm}$ in diameter and $2 \mathrm{~mm}$ thick were prepared from a hybrid-filled resin composite (Te-Econom plus, Ivoclar-Vivadent, Schaan, Liechtenstein) in a custom made Teflon mould. The first increment was placed and light cured for 20 seconds then the second increment was placed. To create a smooth flat surface, the composite was compressed with a glass slide and excess material extruded. The composite was light polymerized with a light-emitting diode device (LED) (LEDEXTMWL-070, Dentmate Technology Co., LTD. New Taipei City, Taiwan) at an intensity of $1000 \mathrm{~mW} / \mathrm{cm}^{2}$ for 20 seconds through the glass plate at a 90 degree angle to the top of the surface. Then, the glass slide was removed and the composite was light polymerized for additional 10 seconds. 
Egyptian

Orthodontic Journal

\section{Aging of the resin composite specimens in vitro}

Specimens were aged by immersion in distilled water at room temperature for 4 weeks.

\section{Mounting the discs in cold cure acrylic resin}

A custom made copper mould was used for mounting the RC discs in cold cure acrylic resin. A hollow cap with a hole diameter $1 \mathrm{~mm}$ larger than discs was used as a guide for placing the discs in the center of the acrylic resin cylinders.

\section{Composite surface treatment}

The specimens were randomly divided into four groups of 15 specimens each according to the following surface treatment methods:

Group 1: Control with no surface treatment.

Group 2: 99\% acetone solution (El-Nasr Pharmaceutical Chemicals Co., Cairo, Egypt.) was used to coat the surface of the composite for 1 minute. The surface then was lightly dried with compressed oil-free air.

Group 3: The disc surface was treated with cold-cured methylmethacrylate monomer (Acrostone cold cure, Acrostone, Cairo, Egypt) for 3 minutes, the surface then was lightly dried with compressed oil-free air.

Group 4: The discs surfaces were roughened at high speed with a $1 \mathrm{~mm}$ round diamond bur (Horico, Berlin, Germany) normal grit with grain size $\sim 80-120 \mu \mathrm{m}$ under water cooling, the rotating bur was passed over the composite surface three times.

\section{Bonding procedure}

The bracket bonding procedures was carried out by one investigator. A thin layer of bonding agent (Ortho solo, Ormco, California, USA.) was applied on the treated composite surface, then adhesive composite resin paste (Grēngloo, Ormco, California, USA.) was placed on the bracket base and the bracket was placed at the center of composite disc and subjected to a $300 \mathrm{~g}$ compressive force using a force gauge (Correx, Bern, 
Egyptian

Orthodontic Journal

Switzerland) for 10 seconds. Excess adhesive was removed using a sharp scaler. Polymerization for 20 seconds from the occlusal side then 20 seconds from the mesial side of the brackets using the LED curing light was performed. Then, all specimens were stored in distilled water for 1 week.

\section{Shear bond strength testing}

Using a universal testing machine (Comten industries, Inc. Florida, USA), an occluso-gingival load was applied to the bracket, producing a shear force at the bracket-composite interface. Shear bond strengths was measured at a crosshead speed of $1 \mathrm{~mm} / \mathrm{minute}$. The maximum load necessary to debond the bracket was recorded in Newtons and converted to megapascals (MPa) as a ratio of Newtons to surface area of the bracket base. The surface area of the bracket was 11.97 as given by the manufacturer.

\section{Adhesive remnant examination}

After debonding, the brackets bases and corresponding RC discs were examined under stereomicroscope (Olympus SZ-CTV, japan) with a color video camera (Panasonic, WV-CP230/G, Germany) at 10X magnification. The area of adhesive remnants was calculated using micro image processing (MIP) (Soft Imaging System SIS, analysis, Gmbh, Germany) software. Afterwards, each disc was assessed using modified adhesive remnant index (ARI) ${ }^{26}$ However, two new scores were added to evaluate the cohesive failure within the restorative composite. The adhesive remnant index scores were given based on the following criteria:

Score 1= All adhesive remained on RC surface with distinct impression of the bracket mesh

Score $2=$ More than $90 \%$ and less than $100 \%$ of adhesive remained on the RC surface.

Score $3=$ More than $10 \%$ but less than $90 \%$ of the adhesive remained on the RC surface.

Score $4=$ Less than $10 \%$ of adhesive remained on the RC surface.

Score $5=$ No adhesive remained of the RC surface.

Score $6=$ Complete cohesive fracture in the restorative composite. (Figure 1).

Volume 48-December 2015 
Egyptian

Orthodontic Journal

Score $7=$ Minimal cohesive fracture of the restorative composite with all the remaining adhesive left on the RC surface. (Figure 2).

\section{Statistical analysis of data}

Comparison between the SBS of different studied groups was analyzed using F-test (1-way analysis of variance (ANOVA)). Adhesive remnant index scores of different groups were compared using Chisquare test with Monte Carlo correction. Significance of the obtained results was judged at the $5 \%$ level $(\mathrm{P} \leq 0.05)$.

\section{RESULTS}

\section{Shear bond strength}

The results of 1-way ANOVA showed no statistical significant difference among the 4 groups where $\mathrm{P}=0.564$. Descriptive analysis and analytical statistics of SBS of the four groups is shown in (Table 1).

\section{Adhesive remnant index}

The result of Chi-square test with Monte Carlo correction for ARI scores between the four groups showed a statistically significant difference $\left({ }^{\mathbf{M C}} \mathbf{p}=0.039\right)$. Chi square comparisons of ARI scores showed statistically significant difference only between group 1 (control) with groups 3 (MMA monomer) ( $\left.\mathrm{P}_{1}=0.003\right)$ and group 4 (diamond bur) $\left(\mathrm{P}_{1}=\right.$ 0.046). Descriptive analysis and analytical statistics of ARI scores of the four studied groups is shown in (Table 2).

Table (1): Descriptive analysis and analytical statistics of SBS of the four studied groups.

\begin{tabular}{|c|c|c|c|c|c|c|c|}
\hline \multirow[t]{2}{*}{ Group } & \multirow[t]{2}{*}{$\mathbf{n}$} & \multicolumn{5}{|c|}{ SBS in MegaPascals } & \multirow[b]{2}{*}{$\begin{array}{c}F \text { and } P \\
\text { value }\end{array}$} \\
\hline & & Mean & $\begin{array}{l}\text { Standard } \\
\text { deviation }\end{array}$ & Minimum & Maximum & Median & \\
\hline 1 & 15 & 10.30 & 4.62 & 4.05 & 18.50 & 10.18 & \multirow{4}{*}{$\begin{array}{l}F=0.687 \\
P=0.564\end{array}$} \\
\hline 2 & 15 & 11.41 & 4.82 & 4.43 & 20.43 & 10.50 & \\
\hline 3 & 15 & 11.04 & 4.25 & 5.90 & 20.14 & 10.10 & \\
\hline 4 & 15 & 12.65 & 4.59 & 5.57 & 20.89 & 11.55 & \\
\hline
\end{tabular}

$\mathrm{F}: \mathrm{F}$ test (ANOVA) 
Egyptian

Orthodontic Journal

Table (2): Descriptive and statistical comparison between the four studied groups according to ARI score

\begin{tabular}{|c|c|c|c|c|c|c|c|c|c|c|}
\hline & \multicolumn{2}{|c|}{$\begin{array}{c}\text { Group } 1 \\
(n=15)\end{array}$} & \multicolumn{2}{|c|}{$\begin{array}{c}\text { Group } 2 \\
(n=15)\end{array}$} & \multicolumn{2}{|c|}{$\begin{array}{c}\text { Group } 3 \\
(n=15)\end{array}$} & \multicolumn{2}{|c|}{$\begin{array}{c}\text { Group } 4 \\
(n=15)\end{array}$} & \multirow{2}{*}{$\chi^{2}$} & \multirow[t]{2}{*}{${ }^{\mathrm{MC}} \mathbf{p}$} \\
\hline & No. & $\%$ & No. & $\%$ & No. & $\%$ & No. & $\%$ & & \\
\hline \multicolumn{11}{|l|}{ ARI score } \\
\hline 1 & 2 & 13.3 & 4 & 26.7 & 8 & 53.3 & 7 & 46.7 & \multirow{7}{*}{$21.915^{*}$} & \multirow{7}{*}{$0.039^{*}$} \\
\hline 2 & 2 & 13.3 & 1 & 6.7 & 0 & 0.0 & 1 & 6.7 & & \\
\hline 3 & 1 & 6.7 & 1 & 6.7 & 1 & 6.7 & 2 & 13.3 & & \\
\hline 4 & 0 & 0.0 & 0 & 0.0 & 1 & 6.7 & 0 & 0.0 & & \\
\hline 5 & 0 & 0.0 & 0 & 0.0 & 0 & 0.0 & 0 & 0.0 & & \\
\hline 6 & 9 & 60.0 & 3 & 20.0 & 1 & 6.7 & 2 & 13.3 & & \\
\hline 7 & 1 & 6.7 & 6 & 40.0 & 4 & 26.7 & 3 & 20.0 & & \\
\hline$p_{1}$ & & & \multicolumn{2}{|c|}{0.073} & \multicolumn{2}{|c|}{$0.003^{*}$} & \multicolumn{2}{|c|}{$0.046^{*}$} & & \\
\hline $\mathbf{P}_{2}$ & & & & & \multicolumn{2}{|c|}{0.499} & \multicolumn{2}{|c|}{0.688} & & \\
\hline p3 & & & & & & & \multicolumn{2}{|c|}{1.000} & & \\
\hline
\end{tabular}

$\chi^{2}$ : Chi square test

MC: Monte Carlo test

$\mathrm{p}_{1}$ : $\mathrm{p}$ value for comparing between group I and each other group

$\mathrm{p}_{2}: \mathrm{p}$ value for comparing between group II with group III and IV

$\mathrm{p}_{3}: \mathrm{p}$ value for comparing between group III and IV

$*$ : Statistically significant at $\mathrm{p} \leq 0.05$
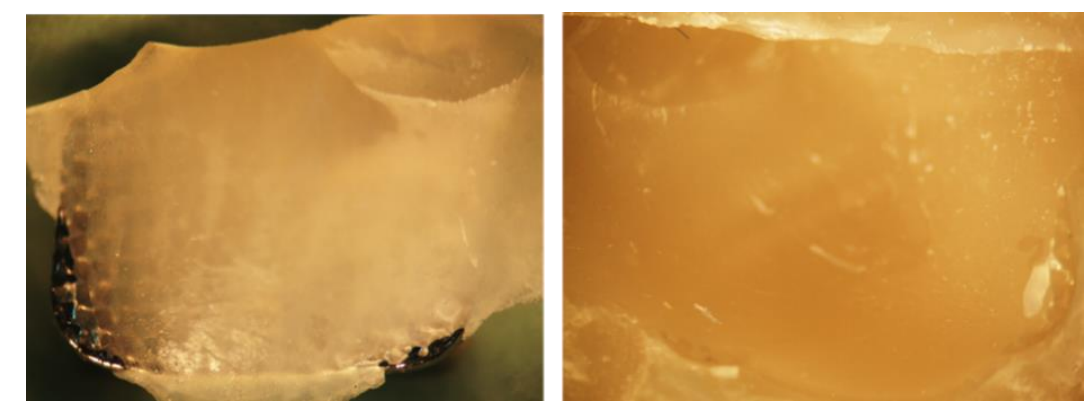

Figure 1: ARI score 6, complete cohesive fracture in the restorative composite. (A) (Magnification X10).

Volume 48-December 2015 
Egyptian

Orthodontic Journal
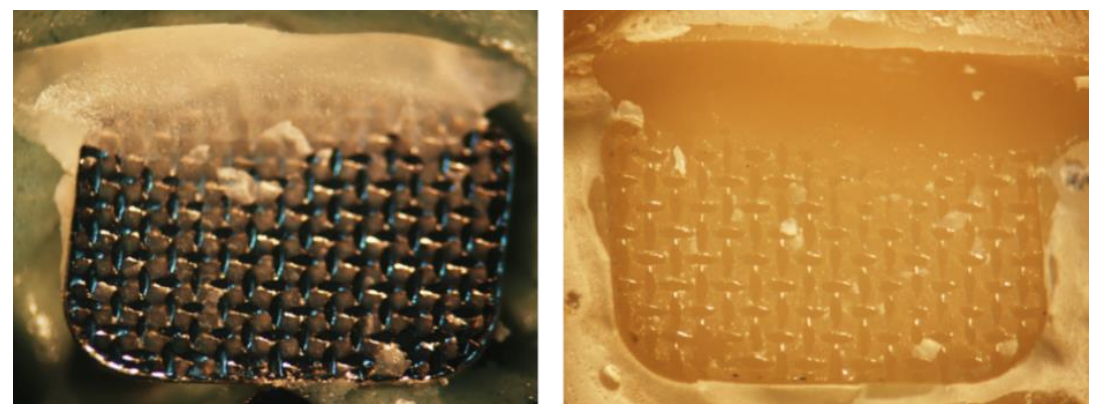

Figure 2: ARI score 7, minimal cohesive fracture of the restorative composite with all the remaining adhesive left on the RC surface. (Magnification X10).

\section{DISCUSSION}

In the present study, The null hypothesis of this study was accepted as no significant difference in SBS was found between any of the groups with SBS of control group $10.30 \pm 4.62 \mathrm{MPa}$, with MMA monomer $11.41 \pm 4.82 \mathrm{MPa}$, with acetone $11.04 \pm 4.25 \mathrm{MPa}$ and with roughening with a diamond bur $12.65 \pm 4.59 \mathrm{MPa}$. However, these values are considered clinically acceptable by Newman ${ }^{27}$ and Reynolds ${ }^{13}$. This can be attributed to factors including the surface treatment, use of bonding agent, aging protocol

\section{Surface treatment}

In this study the control group yielded a clinically acceptable SBS. This comes in acceptance with Newman et al. ${ }^{28}$ who reported no significant difference in SBS of brackets bonded to natural teeth and RC. The surface treatment applied to both groups was phosphoric acid which was reported $^{8,29}$ not to affect the RC. Chunhacheevachaloke and Tyas ${ }^{11}$ also reported that there was no significant difference between SBS of brackets bonded to unroughened and roughened RC surfaces. The results of the present study, however, contradicts the results of other studies showed significantly reduced SBS of control groups. ${ }^{21,30}$ This can be explained by a high SBS of controls in the present study. The factors that caused high SBS of the control group will be discussed in details later.

De Almeida et al. ${ }^{31}$ used MMA monomer as a surface treatment for 180 seconds followed by bonding agent for bonding metallic brackets to 
Egyptian

Orthodontic Journal

acrylic resin and reported a SBS of $9.67 \mathrm{MPa}$. this was explained by the theory that application of MMA monomer can copolymerize with the composite material as the polymerization process of MMA and Bis-GMA follow a similar pattern of activation and cross-linking, because the reactive methacrylate groups of the molecules are similar. ${ }^{24} \mathrm{MMA}$ also appears to induce swelling in the denture teeth and induce micromechanical bond. ${ }^{25}$ In this study, the aim of using MMA monomer was to examine if wetting the RC surface with MMA monomer can affect the $\mathrm{RC}$ surface in the same manner. However, this theory was rejected.

Balkenhol et al. ${ }^{32}$ reported that repair liquids based on Bis-GMA/TEGDMA mixed with acetone are best suited for conditioning the aged surfaces of temporary crown and bridge materials. Acetone in the current study did not provide an additional advantage in bonding orthodontic brackets to RC surface. This agrees with Hamano et al. ${ }^{33,34}$. as the dentin bonding agent was not mixed with acetone instead the acetone was applied separately similar to Hamano et al. ${ }^{33}$

There was an agreement ${ }^{15,21,35}$ that mechanical methods of surface treatment provided a significant increase in bond strength in composite to composite adhesion. According to Bayram et al. ${ }^{21}, \mathrm{Al}_{2} \mathrm{O}_{3}$ particle abrasion and roughening with bur resulted in the highest SBS. On the other hand, diamond bur group yielded a significantly reduced mean SBS of (2.2 MPa, $25.3 \mathrm{~N})$ in Viwattanatipa et al. ${ }^{29}$ study. According to SEM photomicrographs ${ }^{35}$, the application of a diamond bur creates macro- and micro-retentive areas. However, it damages the surface characteristics of the restoration. In addition, patterns of roughening are different among studies and cannot be standardized. It can even weaken el SBS ${ }^{29}$ as it will increase the thickness of bonding agent. In the present study roughening with a diamond bur did not significantly increase the SBS.

Bishara, Ajlouni and Oonsombat ${ }^{36}$ highlighted the importance of bonding agent in bonding brackets to $\mathrm{RC}$ and reported that application of primer and the type of primer are crucial factors in bonding to RC surfaces. This can explain why, in this study, all the studied groups had a high bond strength as bonding agent was applied in all groups. 
Egyptian

Orthodontic Journal

\section{Aging protocol}

In laboratory studies, aging of composite resins has been simulated by storage in water $^{37}$, citric acid immersion ${ }^{38}$, or subjecting them to thermocycling. ${ }^{39}$ In this study, the choice of aging protocol was based on Rinastiti et al. ${ }^{40}$ and Anjum et al. ${ }^{41}$ studies that showed that storage of specimens in water for 4 weeks significantly reduce the bond strength of repaired composite in comparison with control. However, the high bond strength of control group suggests that water immersion for 4 weeks was insufficient for aging the $\mathrm{RC}$ in comparison to thermo-cycling. Demirtas et al. ${ }^{35}$ recommended thermo-cycling for artificial ageing when testing SBS of materials is intended.

\section{Adhesive remnant index (ARI)}

Unlike the SBS, which determines the strength of bond for the whole adhesion complex at all interfaces, the ARI determines the site of failure that provides information about the strength of bond at different interfaces within the same sample. ${ }^{42}$ In general, when testing SBS the failure occurs at the weakest interface. Previous studies ${ }^{11,43}$ reported a high frequency of cohesive RC fracture and modified the ARI accordingly. Also, in this study, 2 different patterns of cohesive failure were found. Complete cohesive failure occurred in all studied groups with the highest frequency of $60 \%$ in the control group and the result was significant in comparison with the MMA monomer group and diamond bur group. The weak adhesion at bracketladhesive interface especially in roughening with bur group can be explained by 3 factors : 1) the weak micro-mechanical retention achieved by the mesh based brackets used in the present study ${ }^{44,45}$ 2) The slight concavity in bracket base opposed to the flat surface of $\mathrm{RC}$ discs 3) Bur roughening make grooves in $\mathrm{RC}$ surface causing concavities and damage the surface structure. Last 2 factors increase thickness of the adhesive resin thus, weakens the bond at bracketladhesive interface. This can explain why in the current study, the statistical analysis revealed insignificant difference in SBS while the results of the ARI were statistically significant. 
Egyptian

Orthodontic Journal

\section{CONCLUSION}

Surface treatment with acetone, MMA monomer or roughening with diamond bur does not affect the SBS of RC stored in water for 4 weeks.

\section{REFERENCES}

1. Newman GV, Facq JM. The effects of adhesive systems on tooth surfaces Am J Orthod. 1971 Jan; 59(1):67-75.

2. Newman GV, Snyder WH, Wilson CE. Acrylic adhesives for bonding attachments to tooth surfaces. Angle orthod. 1968;38:12-18

3. McKiernan EM, McKiernan F, Jones ML. Psychological profiles and motives of adults seeking orthodontic treatment. Int J Adult Orthodon Orthognath Surg. 1992; 7(3):187-198.

4. Gottlieb EL, Nelson AH, Vogels DS. 1990 JCO study of orthodontic diagnosis and treatment procedures. Part 1: Results and trend. J Clin Orthod 1991; 25:145-156.

5. Downer MC. The 1993 national survey of children's dental health: a commentary on the preliminary report. Br Dent J. 1994 Mar 19; 176(6):209-214.

6. Boyer DB, Chan KC, Reinhardt JW. Build-up and repair of light-cured composites: bond strength. J Dent Res. 1984 Oct; 63(10):1241-1244.

7. Dhuru V B, Lloyd C H. The fracture toughness of repaired composite. J Oral Rehabil. 1986 Sep; 13(5):413-421.

8. Kao E C, Pryor H G, Johnston W M. Strength of composites repaired by laminating with dissimilar composites. J Prosthet Dent. 1988 Sep; 60(3):328-333.

9. Chiba K, Hosoda H, Fusayama T. The addition of an adhesive composite resin to the same material: bond strength and clinical techniques. J Prosthet Dent. 1989 Jun; 61(6):669-675.

10. Mizrahi B. A technique for simple and aesthetic treatment of anterior tooth wear. Dent Update. 2004 Mar; 31(2):109-114. 
Egyptian

Orthodontic Journal

11. Chunhacheevachaloke E, Tyas MJ. Shear bond strength of ceramic brackets to resin-composite surfaces. Aust Orthod J. 1997 Oct; 15(1):10-15.

12. Newman GV, Newman RA, Sun BI, Ha JL, Ozsoylu SA. Adhesion promoters, their effect on the bond strength of metal brackets. Am J Orthod Dentofacial Orthop. 1995 Sep; 108(3):237-241.

13. Reynolds IR. A review of direct orthodontic bonding. Br J Orthod 1975; 2:171-178.

14. Gillis I, Redlich M. The effect of different porcelain conditioning techniques on shear bond strength of stainless steel brackets. Am J Orthod Dentofacial Orthop. 1998 Oct; 114(4):387-392.

15. Eslamian L, Borzabadi-Farahani A, Mosavian N, Ghasemi A. The effects of various surface treatments on the shear bond strengths of stainless steel brackets to artificially aged composite restorations. Aust Orthod J. 2011 May; 27(1):28-32.

16. Eslamian L, Ghassemi A, Amini F, Jafari A, Afrand M. Should silane coupling agents be used when bonding brackets to composite restorations? An in vitro study. Eur J Orthod. 2009 Jun;31(3):266-270

17. Schwartz RE, Tyas MJ, West VC. Current note No. 71. The bonding of orthodontic brackets to composite resin surfaces. Aust Dent J. 1990 Oct; 35(5):472-3.

18. Bishara S E, Fehr D E, Jakobsen J R. A comparative study of debonding strengths of different ceramic brackets, enamel conditioners, and adhesives. Am J Orthod Dentofacial Orthop. 1993 Aug; 104(2):170-9.

19. Magni E, Ferrari M, Papacchini F, Hickel R, Ilie N. Influence of ozone application on the repair strength of silorane-based and ormocer-based composites. Am J Dent. 2010 Oct; 23(5):260-4.

20. Cho SD, Rajitrangson P, Matis BA, Platt JA. Effect of Er,Cr:YSGG laser, air abrasion, and silane application on repaired shear bond strength of composites. Oper Dent. 2013 May-Jun; 38(3):E1-9.

21. Bayram M, Yesilyurt C, Kusgöz A, Ulker M, Nur M. Shear bond strength of orthodontic brackets to aged resin composite surfaces: effect of surface conditioning. Eur J Orthod. 2011 Apr; 33(2):174-9. 
Egyptian

Orthodontic Journal

22. Lucena-Martín C, González-López S, Navajas-Rodríguez de Mondelo JM. The effect of various surface treatments and bonding agents on the repaired strength of heat-treated composites. J Prosthet Dent. 2001 Nov; 86(5):481-488.

23. Vergani CE, Machado AL, Giampaolo ET, Pavarina AC. Effect of surface treatments on the bond strength between composite resin and acrylic resin denture teeth. Int J Prosthodont. 2000 Sep-Oct; 13(5):383-386.

24. Cook WD, Beech DR, Tyas MJ. Structure and properties of methacrylate based dental restorative materials. Biomater1985 Nov; 6(6): 362-368.

25. Papazoglou E, Vasilas AI. Shear bond strengths for composite and autopolymerized acrylic resins bonded to acrylic resin denture teeth. J Prosthet Dent. 1999 Nov; 82(5):573-578.

26. Bishara SE, Ostby AW, Laffoon JF, Warren JJ. The effect of modifying the self-etchant bonding protocol on the shear bond strength of orthodontic brackets. Angle Orthod 2007; 77(3):504-508

27. Newman GV. Epoxy adhesives for orthodontic attachments, progress report. Am J Orthod. 1965; 51:901-912.

28. Newman SM, Dressler KB, Grenadier MR. Direct bonding of orthodontic brackets to esthetic restorative materials using a silane. Am J Orthod. 1984 Dec; 86(6):503-6.

29. Viwattanatipa N, Jermwiwatkul W, Chintavalakorn R, Nanthavanich $\mathrm{N}$. The effect of different surface preparation techniques on the survival probabilities of orthodontic brackets bonded to nanofill composite resin. J Orthod. 2010 Sep; 37(3):162-73.

30. Brunharo IH, Fernandes DJ, de Miranda MS, Artese F. Influence of surface treatment on shear bond strength of orthodontic brackets. Dental Press J Orthod. 2013 May-Jun; 18(3):54-62.

31. De Almeida JX, Deprá MB, Marquezan M, Retamoso LB, Tanaka O. Effects of surface treatment of provisional crowns on the shear bond strength of brackets. Dental Press J Orthod. 2013 Jul-Aug; 18(4):29-34. 
Egyptian

Orthodontic Journal

32. Balkenhol M, Michel K, Stelzig J, Wostmann B. Repairability of cross-linked biopolymers. J Dent Res 2009; 88:152-157.

33. Hamano N, Chiang YC, Nyamaa I, Yamaguchi H, Ino S, Hickel R, Kunzelmann KH. Repair of silorane-based dental composites: influence of surface treatments. Dent Mater. 2012 Aug; 28(8):894-902.

34. Hamano N, Chiang YC, Nyamaa I, Yamaguchi H, Ino S, Hickel R, Kunzelmann KH. Effect of different surface treatments on the repair strength of a nanofilled resin-based composite. Dent Mater J. 2011; 30(4):537-545.

35. Demirtas HK, Akin M, Ileri Z, Basciftci FA. Shear-bond-strength of orthodontic brackets to aged nano-hybrid composite-resin surfaces using different surface preparation. Dent Mater J. 2015; 34(1):86-90.

36. Bishara SE, Ajlouni R, Oonsombat C. Bonding Orthodontic Brackets to Composite Using Different Surface Preparations and Adhesive/Primers: A Comparative Study World J Orthod 2003; 4:343-347.

37. Cesar P, Meyer Faara P, Miwa Caldart R, Gastaldoni Jaeger R, da Cunha RF. Tensile bond strength of composite repairs on Artglass using different surface treatments. Am J Dent. 2001; 14:373-377

38. Özcan M, Barbosa S, Melo R, Galhano G, Bottino M. Effect of surface conditioning methods on the microtensile bond strength of resin composite to composite after aging conditions. Dent Mater 2007; 23:1276-1282

39. Hakimeh S, Vaidyanathan J, Houpt ML, Vaidyanathan TK, Von Hagen S. Microleakage of compomer class V restorations: effect of load cycling, thermal cycling, and cavity shape differences. J Prosthet Dent 2000; 83:194-203

40. Rinastiti M, Özcan M, Siswomihardjo W, Busscher H J. Effects of surface conditioning on repair bond strengths of non-aged and aged microhybrid, nanohybrid, and nanofilled composite resins. Clin Oral Investig. 2011 Oct; 15(5):625-633.

41. Anjum A, Matin K, Uchida R, Tagami J. influence of aging on direct resin composite repair bond. Int Chin J Dent 2008; 8: 53-61. 
Egyptian

Orthodontic Journal

42. Carstensen W. Effect of reduction of phosphoric acid concentration on the shear bond strength of brackets. . Am J Orthod Dentofacial Orthop 1995; 108:274-277

43. Lai PY, Woods MG, Tyas MJ. Bond strengths of orthodontic brackets to restorative resin composite surfaces. Aust Orthod J. 1999 Apr; 15(4):235-245.

44. Olivier S, EL Alam R, Francis C, Guy C. Comparison of bond strength between simple foil mesh and laser-structured base retention brackets. Am J Orthod Dentofacial Orthop 2002; 122:260-6.

45. Mehta OP, Saini S, Dahiya A. Comparative evaluation of shear bond strength of different bracket base designs in dry and wet environments. J Ind Soc Ped Prev Dent 2008; 26(7):104-8. 